\title{
Rendimiento productivo y composición corporal de pollos alimentados con harina de poroto mucuna (Stizolobium deeringianum)
}

\author{
Fernández, R. .' Revidatti, F.'; Sindik, M. ${ }^{1}$; Sanz, P.'; Sandoval, G. ${ }^{2}$ \\ ${ }^{1}$ Cátedra Producción de Aves, ${ }^{2}$ Cátedra Bioquímica, Facultad de Ciencias Veterinarias, UNNE, \\ Sargento Cabral 2139, Corrientes (3400), Tel/Fax: 54-379-4425753. \\ E-mail: granja@vet.unne.edu.ar.
}

\begin{abstract}
Resumen
Fernández, R.; Revidatti, F.; Sindik, M.; Sanz, P.; Sandoval, G.: Rendimiento productivo y composición corporal de pollos alimentados con harina de poroto mucuna (Stizolobium deeringianum). Rev. vet. 24: 2, 102-106, 2013. Las leguminosas tropicales ricas en proteínas son de interés por su valor nutricional y bajo costo, aunque su uso debe ser precedido por estudios biológicos y económicos que permitan establecer su eficiencia. En el presente trabajo se estudió el efecto de la incorporación de harina de poroto mucuna (Stizolobium deeringianum) en reemplazo parcial de la soja, sobre las variables productivas y rendimiento a la faena de pollos Campero INTA. La dieta control fue elaborada en base a maíz, soja y una premezcla para alimento terminador. En la dieta de prueba, el poroto mucuna tratado con $\mathrm{Ca}(\mathrm{OH})_{2}$ al $1 \%$ reemplazó el 30\% de la harina de soja. El análisis comparativo se realizó mediante ANOVA en un arreglo factorial, con el sexo y tipo de alimento como efectos principales. Se observaron diferencias estadísticamente significativas $(\mathrm{p}<0,05)$ para ganancia de peso, con valores de $1.385,27 \pm 281,52 \mathrm{~g}$ para los pollos alimentados con la dieta control y $1.258,63 \pm 365,27 \mathrm{~g}$ para la de prueba. El consumo de alimento demostró una diferencia numérica a favor de los controles $(410 \mathrm{~g})$, aunque el grado de significancia solo manifestó una tendencia a la separación estadística $(\mathrm{p}=0,08)$. Las variables de composición corporal no mostraron diferencias estadísticas entre los tratamientos. La inclusión de poroto mucuna tratado con solución alcalina produjo disminución de la ganancia de peso en pollos Campero INTA en fase de terminación. Se considera necesario profundizar los estudios sobre los métodos de eliminación de los factores antinutricionales, de tal manera que permitan la utilización de este tipo de alimento.
\end{abstract}

Palabras clave: pollo, alimentación, dieta, eficiencia.

\begin{abstract}
Fernández, R.; Revidatti, F.; Sindik, M.; Sanz, P.; Sandoval, G. Productive performance and body composition of chickens fed mucuna bean meal (Stizolobium deeringianum). Rev. vet. 24: 2, 102-106, 2013. The use of tropical legumes rich in protein is of interest for its nutritional value and low cost, but must be preceded by biological and economic studies to establish their efficiency. The effects of mucuna (Stizolobium deeringianum) bean flour incorporation as partial replacement of soybean in the finisher food on productive performance and yielding at slaughter in Campero INTA chickens were studied. The control diet was developed based on corn, soybeans and finisher feed premix. In the trial diet, mucuna bean treated with $\mathrm{Ca}(\mathrm{OH})_{2} 1 \%$ replaced $30 \%$ of soybean. The comparative analysis was performed using a factorial ANOVA, with sex and type of food as main effects. The weight gain of the chickens fed with the control diet was statistically higher than the values of mucuna diet with 1.385,27 $\pm 281,52 \mathrm{~g}$ and $1.258,63 \pm 365,27 \mathrm{~g}$ respectively. The feed intake of the control group showed a numerical difference, although the degree of significance only showed a trend towards statistical separation $(p=0,08)$. Body composition variables showed no statistical differences between treatments. Mucuna bean treated with alkaline solution causes a decrease of weight gain in Campero INTA chickens during the fattening phase. Further studies are necessary regarding the removal of anti-nutritional factors that may enable the practical use of new types of foods.
\end{abstract}

Key words: chicken, food, diet, efficiency. 


\section{INTRODUCCIÓN}

La producción avícola depende de algunos insumos críticos para su desarrollo, entre los cuales se destaca la alimentación. Tanto a escala industrial como en la alternativa de traspatio, los altos costos de las materias primas tradicionales han llevado a la necesidad de investigar nuevas fuentes de proteína vegetal en reemplazo de la soja ${ }^{9}$.

La utilización de otras leguminosas ricas en proteínas, como el poroto mucuna, resultan de interés por su valor nutricional y bajo costo, aunque deben ser precedida por estudios biológicos y económicos que permitan establecer su eficiencia ${ }^{15}$. El contenido de proteína de estos porotos se encuentra en valores que van de 23,5 a $38,4 \%{ }^{8}$, con una digestibilidad que oscila entre el 84,2 y $85,5 \%$, destacándose su alto contenido en lisina, atributo que les otorga gran valor nutricional para dietas de monogástricos ${ }^{2}$, aunque presentan deficiencias de metionina y cistina ${ }^{8}$.

Estos porotos contienen metabolitos activos que pueden producir efectos tóxicos sobre los animales, denominados genéricamente factores antinutricionales (FAN), producidos por las plantas como mecanismos defensivos al ataque de distintos agentes. Entre ellos se destaca la presencia de L-Dopa, señalada como el principal componente antinutricional por su concentración, efectos adversos y dificultades en su eliminación ${ }^{17}$. Otros componentes presentes como fenoles, taninos, lectinas y ácido fítico ${ }^{14}$ también deprimen el consumo e impiden la digestión, absorción y utilización de los nutrientes ${ }^{1}$.

Los altos contenidos de FAN en estas leguminosas han llevado a la necesidad de utilizar métodos físicos y químicos para minimizarlos y obtener un producto de óptima calidad nutricional ${ }^{3}$. La hidratación alcalina con $\mathrm{Ca}(\mathrm{OH})_{2}$ al $1 \%$ en una proporción 1:10 durante 24 $\mathrm{h}$ y posterior secado en estufa a $55^{\circ} \mathrm{C}$ por $48 \mathrm{~h}$, ha demostrado ser efectiva en una reducción adecuada de los FAN en estos porotos ${ }^{12}$.

En el presente trabajo se estudió el efecto de la incorporación de harina de porotos mucuna (Stizolobium deeringianum), tratado mediante hidratación alcalina en reemplazo parcial de la soja en el alimento terminador, sobre las variables productivas y rendimiento de cortes valiosos en pollos Campero INTA.

\section{MATERIAL Y MÉTODOS}

Sujetos experimentales. El ensayo se llevó a cabo en el aviario de la Facultad de Ciencias Veterinarias de la Universidad Nacional del Nordeste (Corrientes, Argentina) entre el 22 de enero y el 16 de abril de 2013, bajo un protocolo de trabajo aprobado por el Comité de Bioética de esta Institución. Se dispuso de un galpón semiabierto de 9 por $15 \mathrm{~m}$, con techo de cinc, paredes de mampostería, extremos cerrados y piso de cemento alisado.
Se utilizó un lote mixto (machos y hembras) de 120 pollos Campero INTA de 1 día de edad, provistos por el Instituto Nacional de Tecnología Agropecuaria (INTA, Corrientes). Los animales de ambos sexos se criaron a piso en forma conjunta, bajo las mismas condiciones ambientales y de manejo hasta el día 42. Desde este momento fue posible separar los sexos de acuerdo a los caracteres sexuales externos y dar inicio al ensayo, seleccionando al azar 40 machos y 40 hembras, que se alojaron en 16 boxes de $1,5 \mathrm{~m}^{2}$ ( 8 para machos y 8 para hembras) a razón de 5 aves por compartimiento.

En cada box el agua fue provista con bebedero de plato con recipiente invertido de 4 litros, y el alimento con comederos tolva de $3 \mathrm{~kg}$ de capacidad. Durante el período del ensayo se registraron temperaturas máximas con un promedio de $28,8^{\circ} \mathrm{C}$ y mínimas con promedios de $20^{\circ} \mathrm{C}$; la humedad relativa ambiente osciló entre 59 y $86 \%$. Al inicio del ensayo las aves registraron un peso promedio de $1.236,56 \pm 167,13 \mathrm{~g}$.

Diseño experimental. Para estudiar el efecto de la utilización de harina de poroto mucuna (Stizolobium deeringianum) en reemplazo de un $30 \%$ de la harina de soja del alimento terminador, sobre las variables productivas y rendimiento de cortes valiosos en pollos Campero INTA, se empleó un arreglo factorial, con el sexo y la dieta como fuentes de variación.

La variable independiente estuvo constituida por dos tipos de dietas suministradas ad libitum desde el día 42 al 84 de vida. La dieta control se elaboró en base a maíz, soja y una premezcla para balanceado de parrilleros; en la dieta de prueba el poroto mucuna reemplazó parcialmente a la harina de soja. Para la disminución de los FAN, el poroto mucuna fue sometido a hidratación alcalina en solución de $\mathrm{Ca}(\mathrm{OH})_{2}$ al $1 \%$ (en relación 1:10 en volumen) durante $24 \mathrm{~h}$ y posterior secado en estufa a $55^{\circ} \mathrm{C}$ durante $48 \mathrm{~h}$, luego molido a un tamaño de $1 \mathrm{~mm}^{9}$.

Muestras de los ingredientes fueron analizadas mediante el procedimiento de la AOAC ${ }^{6}$. La composición proximal de la harina de poroto mucuna tratado demostró que contenía 27,56\% de proteína bruta, 3,09 de extracto etéreo, $5,92 \%$ de fibra bruta y un valor de energía metabolizable de $3.360 \mathrm{kcal} / \mathrm{kg}$. Cada dieta fue suministrada a 4 boxes de machos y 4 de hembras, constituyendo cada uno una unidad experimental en el ensayo. La composición química de los alimentos se calculó mediante el software Zootec 3.0 y se expone en la Tabla 1.

Variables dependientes. Al final del ensayo se registraron las siguientes variables productivas:

Ganancia de peso (g): obtenida por diferencia entre el promedio de peso corporal al final y el promedio de peso corporal al inicio del ensayo en cada box.

Consumo de alimento (g): total de alimento consumido durante el ensayo en cada box divido la cantidad de aves. 
Tabla 1. Composición de las dietas de terminación suministradas en el ensayo.

\begin{tabular}{lcc}
\hline & $\begin{array}{c}\text { alimento } \\
\text { control }\end{array}$ & $\begin{array}{c}\text { alimento } \\
\text { mucuna }\end{array}$ \\
\hline composición porcentual & 65 & \\
maíz (\%) & 30 & 65 \\
soja (\%) & 0 & 21 \\
mucuna (\%) & 5 & 9 \\
premezcla (\%) & & 5 \\
composición calculada & 88,18 & 88,42 \\
materia seca (\%) & 17,84 & 16,51 \\
proteína bruta (\%) & 5,12 & 4,69 \\
extracto etéreo (\%) & 3,36 & 3,32 \\
fibra bruta (\%) & 1,09 & 1,08 \\
calcio (\%) & 0,48 & 0,5 \\
fósforo (\%) & 2.936 & 3.013 \\
energía metaboliz. (kcal/kg) & &
\end{tabular}

Conversión alimenticia: relación entre alimento consumido y ganancia de peso.

Las aves fueron sacrificadas por yugulación previa insensibilización mediante el método descrito por Mota Rojas ${ }^{16}$, para luego obtener las variables:

Rendimiento de canal (\%): se obtuvo dividiendo el peso de la canal (sin cabeza, patas ni vísceras) por el peso corporal y luego multiplicando por 100 .

Rendimiento de pechuga (\%): luego de separar y pesar la pechuga con hueso, se obtuvo su porcentaje con respecto al peso corporal.

Rendimiento de pata-muslo (\%): luego de separar y pesar la pata muslo, se obtuvo su porcentaje con respecto al peso corporal.

Panículo adiposo (\%): luego de disecar y pesar el panículo adiposo abdominal, se obtuvo su porcentaje con respecto al peso de la canal.

Análisis estadístico. Se calcularon los estadísticos descriptivos media aritmética, desvío estándar, coeficiente de variación, mínimo y máximo, a cada una de las variables dependientes. La distribución de todas las variables se constató mediante el método de Wilk-Shapiro modificado y la homogeneidad de las varianzas mediante el método de Levene. El análisis comparativo se realizó mediante ANOVA en un arreglo factorial, tomándose como efectos principales el sexo y tipo de alimento, considerando límite un nivel de significancia del 5\% ${ }^{19}$. Se analizó la interacción sexo por tratamiento con un nivel de significancia del 5\% y las relaciones entre las variables propuestas para cada factor (correlación de Pearson).

\section{RESULTADOS}

Estadística descriptiva. En la Tabla 2 se observan los valores de la estadística descriptiva obtenidos en pollos Campero INTA al final del período de estudio.

Análisis de la varianza. El resultado del estudio de las interacciones entre los distintos niveles de los factores incluidos en el modelo no fue estadísticamente significativo $(\mathrm{p}>0,05)$, esto permitió analizar los efectos principales dieta y sexo. En la Tabla 3 se exponen los resultados obtenidos para las variables productivas y rendimiento de cortes valiosos para el factor dieta. Tanto en pollos alimentados con dieta control y experimental, la ganancia de peso corporal correlacionó en forma positiva y significativa con el consumo de alimento $\left(r^{2}=0,96 p=0,0001\right)$.

En la Tabla 4 se observan los resultados del análisis de la varianza según el factor sexo para ambos alimentos en conjunto.

\section{DISCUSIÓN}

Dado que no existen procesos ni técnicas estandarizadas para el tratamiento del poroto mucuna, los métodos empleados tienen efectos variables sobre su valor nutricional. Se han investigado distintas técnicas que incluyen remojado en agua o con soluciones alcalinas o ácidas, el calor y la cocción. Los efectos de los procesos sobre la calidad de la materia prima, se expresan tanto en la disponibilidad de nutrientes, como en la concentración de los FAN presentes en el producto final $^{7}$.

En el presente estudio la incorporación de harina del poroto mucuna dio por resultado una disminución del consumo voluntario de alimento $(7,86 \%$ con respecto al control). Aunque la diferencia registrada entre ambos grupos experimentales no alcanzó significancia estadística, reveló una tendencia a favor del grupo control. Esta merma en el consumo de alimento es atribuible al efecto de los FAN residuales sobre la conducta ingestiva de las aves ${ }^{5,13}$. La cocción en solución de $\mathrm{Ca}(\mathrm{OH})_{2}$ al $4 \%$ durante $30 \mathrm{~min}$ y posterior secado a $60^{\circ} \mathrm{C}$ logró minimizar este efecto adverso, obteniéndose resultados similares de consumo para dieta control y mucuna ${ }^{21}$.

Existieron diferencias significativas en la ganancia de peso a favor del grupo control, debidas al mayor consumo de alimento, dada la correlación positiva entre ambas variables. Esta merma en la ganancia de peso en los pollos alimentados con mucuna es atribuible tanto a la deficiencia en metionina del poroto, como a los efectos adversos ocasionadas por los FAN ${ }^{5}$. Pollos alimentados en fase de terminación con dietas incluyendo poroto mucuna hervido durante 20, 40 y 60 minutos, mostraron una ganancia de peso directamente proporcional al tiempo de cocción, atribuible principalmente a la mayor eliminación de L-dopa ${ }^{20}$. Una mayor movilización de estos FAN se logra sumergiendo a los porotos en una solución alcalina de $\mathrm{Ca}(\mathrm{OH})_{2}$ al $4 \%$ durante $24 \mathrm{~h}$; el quebrado previo favorece la exposición al álcali, alcanzando una casi completa eliminación de la L-dopa lo que se ve reflejado en mayor consumo de alimento y ganancia de peso ${ }^{21}$.

No existieron diferencias significativas para la conversión alimenticia. Dietas para pollos parrilleros en etapa de engorde conteniendo 6,12 y $18 \%$ de mu- 
Tabla 2. Estadística descriptiva de las variables productivas y cortes valiosos en los pollos bajo ensayo.

\begin{tabular}{lccccc}
\hline & $\overline{\mathrm{x}}$ & $\mathrm{DE}$ & $\mathrm{CV}$ & mínimo & máximo \\
\hline peso corporal a 84 días $(\mathrm{g})$ & $2.558,50$ & 480,80 & 18,79 & $1.908,00$ & $3.176,00$ \\
ganancia total de peso $(\mathrm{g})$ & $1.321,95$ & 321,75 & 24,34 & 873 & 1746 \\
consumo de alimento $(\mathrm{g})$ & $5.011,44$ & $1.199,04$ & $2.3,93$ & 2.760 & 6.660 \\
conversión alimenticia & 3,8 & 0,27 & 7,22 & 3,16 & 4,24 \\
rendimiento de canal (\%) & 70,57 & 1,69 & 2,39 & 67,38 & 73,53 \\
rendimiento de pechuga (\%) & 18,16 & 2,11 & 11,64 & 13,89 & 21,71 \\
rendimiento pata-muslo (\%) & 20,92 & 1,07 & 5,13 & 19,04 & 22,58 \\
panículo adiposo (\%) & 3,72 & 0,95 & 25,66 & 2,14 & 5,83 \\
\hline
\end{tabular}

$\overline{\mathrm{X}}$ : media aritmética, DE: desvío estándar, CV: coeficiente de variación.

Tabla 3. Análisis de la varianza en pollos alimentados con mucuna versus controles $(n=16)$.

\begin{tabular}{lcccccc}
\hline & \multicolumn{2}{c}{ control } & \multicolumn{2}{c}{ mucuna } & \multirow{2}{*}{$\mathrm{F}$} & $\mathrm{p}$ \\
\cline { 2 - 5 } & $\overline{\mathrm{x}}$ & $\mathrm{DE}$ & $\overline{\mathrm{x}}$ & $\mathrm{DE}$ & & \\
\hline ganancia total de peso (g) & $1.385,27$ & 281,52 & $1.258,63$ & 365,27 & 7,35 & 0,01 \\
consumo de alimento (g) & $5.216,25$ & $1.028,52$ & $4.806,63$ & $1.388,17$ & 3,6 & 0,08 \\
conversión alimenticia & 3,77 & 0,19 & 3,82 & 0,35 & 0,12 & 0,73 \\
rendimiento de canal (\%) & 71,13 & 1,94 & 70,02 & 1,29 & 2,06 & 0,17 \\
rendimiento de pechuga (\%) & 18,19 & 2,55 & 18,13 & 1.75 & 0,01 & 0,94 \\
rendimiento de pata muslo (\%) & 20,86 & 1,18 & 20,98 & 1,03 & 0,05 & 0,81 \\
panículo adiposo (\%) & 3,74 & 0,99 & 3,7 & 0,99 & 0,01 & 0,92 \\
\hline
\end{tabular}

X̄: media aritmética, DE: desvío estándar, F: distribución, p: significancia.

Tabla 4. Análisis de la varianza en cada sexo del conjunto de pollos bajo ensayo.

\begin{tabular}{|c|c|c|c|c|c|c|}
\hline & \multirow{2}{*}{$\begin{array}{c}\text { machos } \\
\bar{x}\end{array}$} & \multicolumn{3}{|c|}{ hembras } & \multirow[b]{2}{*}{$\mathrm{F}$} & \multirow[b]{2}{*}{$\mathrm{p}$} \\
\hline & & DE & $\bar{x}$ & DE & & \\
\hline ganancia total de peso $(\mathrm{g})$ & $1.615,13$ & 108,26 & $1.028,77$ & 116,85 & 157,55 & 0,0001 \\
\hline consumo de alimento (g) & $6.085,88$ & 288,67 & 3.937 & 598,97 & 99,08 & 0,0001 \\
\hline conversión alimenticia & 3,78 & 0,20 & 3,82 & 0,35 & 0,08 & 0,77 \\
\hline rendimiento de canal (\%) & 69,91 & 1,61 & 71,23 & 1,59 & 2,93 & 0,11 \\
\hline rendimiento de pechuga (\%) & 16,87 & 1,96 & 19,45 & 1,39 & 8,56 & 0,01 \\
\hline rendimiento pata-muslo $(\%)$ & 21,39 & 1,00 & 20,46 & 0,99 & 3,27 & 0,09 \\
\hline panículo adiposo $(\%)$ & 3,12 & 0,56 & 4,32 & 0,90 & 9,46 & 0,008 \\
\hline
\end{tabular}

$\overline{\mathrm{X}}$ : media aritmética, DE: desvío estándar, F: distribución, p: significancia.

cuna tostada produjeron disminución en el consumo voluntario de alimento y en la ganancia de peso corporal, aunque en coincidencia con el presente trabajo, no tuvieron efecto en la eficiencia de utilización del alimento ${ }^{13}$. Sin embargo, algunos autores han señalado que al relacionar estos indicadores productivos, la conversión alimenticia puede tener un comportamiento inconsistente ${ }^{11}$.

La composición química de los ingredientes tiene una influencia significativa sobre las características de la canal y calidad de la carne. La dieta puesta a prueba no produjo efectos estadísticamente significativos sobre la composición corporal, expresados como el rendimiento de la canal, cortes valiosos y contenido de grasa abdominal. Dietas para pollos conteniendo mucuna tratado mediante remojado y descascarado, e incluido al 25 y $30 \%$ no registraron diferencias significativas para peso de carcasa ${ }^{4}$. Otros investigadores señalan que el remojado durante 24 horas y posterior cocción durante
60 min logran revertir en gran medida los efectos de los FAN y mejoran el perfil nutricional del alimento y su asimilación, lo que se refleja en un mayor rendimiento de los cortes valiosos ${ }^{20}$.

Los resultados obtenidos permiten confirmar que el factor sexo influye tanto en los resultados productivos, como en los relacionados con el rendimiento de la canal y cortes valiosos. En este ensayo se registraron diferencias significativas para consumo de alimento y ganancia de peso a favor de los machos, observándose una conversión alimenticia similar para ambos sexos. El efecto del factor sexo sobre las variables productivas ha sido señalado en otros estudios, que describen mayor consumo y peso vivo para los machos independientemente del genotipo al cual pertenecen, confirmando que esta característica es propia de la especie y por lo tanto es constatable en distintos contextos productivos y experimentales ${ }^{22}$. 
Se ha verificado que esta diferencia entre sexos se incrementa con la edad independientemente del genotipo de ave estudiada ${ }^{10}$. Asimismo, en coincidencia con trabajos anteriores en estos genotipos aviares, los resultados de rendimiento de la canal no arrojaron diferencias significativas, aunque se observó un mayor porcentaje de pechuga y grasa abdominal en las hembras ${ }^{18}$.

En conclusión, la incorporación de un 9\% de harina de porotos mucuna tratada con solución de hidróxido de calcio al 1\%, lleva a una disminución de la ganancia de peso en pollos Campero INTA en fase de terminación. Se considera necesario profundizar los estudios sobre los métodos de eliminación de los factores antinutricionales, de tal manera que permitan la utilización de este tipo de alimento.

\section{REFERENCIAS}

1. Adebowale Y, Adeyemi A, Oshodi A. 2005. Variability in the physicochemical, nutritional and antinutritional attributes of six Mucuna species. Food Chem 89: 37-48.

2. Adebowale Y, Adeyemi I, Oshodi A, Niranjan K. 2007. Isolation, fractionation and characterization of proteins from Mucuna bean. Food Chem 104: 287-299.

3. Adewale E, Ologhobo D, Gous M. 2007. Influence of processing of mucuna (Mucuna pruriens) and kidney bean (Phaseolus vulgaris) on the performance and nutrient utilization of broiler chickens. J Poult Sci 44: 168-174.

4. Adzitey F, Teye G, Dei H, Okine C. 2010. Effects of processed Mucuna pruriens (var cochinchiensis) beans on carcass characteristics of broiler chicken. Livestock Res Rural Develop 22: 6. On line: http://www.lrrd.org/ lrrd22/6/okin22113.htm.

5. Akinmutimi A, Okwu N. 2006. Effect of quantitative substitution of cooked Mucuna utilis seed meal for soybean meal in broiler finisher diet. Int J Poult Sci 5: 477-481.

6. AOAC. 1990. Official methods of analysis, $15^{\text {th }}$ ed., Publ. Association of Official Analytical Chemists, Virginia, USA.

7. Carew L, Gernat A. 2006. Use of velvet beans, Mucuna spp., as a feed ingredient for poultry: a review. World's Poult Sci J 62: 131-144.

8. Chaparro Acuña SP, Aristizábal, I, Gil J. 2009. Composición y factores antinutricionales de las semillas del género Mucuna. Rev Fac Nal Agr (Medellín, Colombia) 62: 4843-4853.

9. Chaparro Acuña SP. 2009. Efecto de diferentes procesos fisicoquímicos en la reducción de factores antinutricionales de la semilla de vitabosa (Mucuna deeringiana). Tesis Mgt (Fac Cs Agropec Univ Nac Colombia), 81 p.

10. Durán M, Solana J, Cancho M. 1997. Resultados productivos, rendimiento y composición de la canal de dos cruces de pollos de carne criados en libertad. Arch Zootec 46: 239-247.
11. Emenalom O, Udedibie A, Esonu B, Etuk E. 2005. Evaluation of processed velvet vean (Mucuna pruriens) as a feed ingredient in starter diets for broiler chickens. J Poultry Sci 42: 301-307.

12. Gurumoorthi P, Janardhanan K, Myhrman R. 2008. Effect of differential processing methods on L-Dopa and protein quality in velvet bean, an underutilized pulse. LWT Food Sci Technol 41: 588-596.

13. Iyayi E, Taiwo V. 2003. The effect of diets incorporating mucuna (Mucuna pruriens) seed meal on the performance of laying hens and broilers. J Trop \& Subtrop Agr 1: 239246.

14. Janardhanan K, Gurumoorthi P, Pugalenthi M. 2003. Nutritional potential of five accessions of a South Indian tribal pulse, Mucuna pruriens var. utilis. The effect of processing methods on the content of L-Dopa, phytic acid and oligosaccharides. J Trop \& Subtrop Agr 1: 141-152.

15. Lon-Wo E, Cino D. 2000. Aminoácidos sintéticos en la eficiencia de utilización de una fuente proteica (Vigna unguiculata) alternativa para pollos de ceba. Rev Cub Cien Agric 34: 341-346.

16. Mota Rojas D, Maldonado M, Becerril M, Flores S, González M, Alonso M, Camacho D, Ramírez, Cardona A, Morfín L. 2008. Welfare at slaughter of broiler chickens: a review. Int J Poultry Sci 7: 1-5.

17. Mugendi J, Njagi E, Kuria E, Mwasaru M, Mureithi J, Apostolides Z. 2010. Effects of processing methods on the protein quality of mucuna bean (Mucuna pruriens). Afr $J$ Food Agric Nutr \& Dev 10: 14-15.

18. Panno A, Canet Z, Antruejo A, Tersaghi A, Galvagni A, Di Masso R, Font M, Dottavio A. 2004. Relación entre el peso corporal y la proporción de cortes valiosos a la faena en pollos Campero con aporte de genes Cornish por vía materna y/o paterna. Jornadas de Divulgación Técnico-Cientificas, Casilda (Santa Fe, Argentina). On line: http:// veterinarias.e-ducativa.com/upload/jornadas2004.pdf.

19. Steel R, Torrie J. 1988. Bioestadística: principios y procedimientos, McGraw-Hill, México, $622 \mathrm{p}$.

20. Tuleun C, Igba F. 2008. Growth and carcass characteristics of broiler chickens fed water soaked and cooked velvet bean (Mucuna utilis) meal. Afr J Biotech 7: 2676-2681.

21. Ukachukwu S, Szabo N. 2003. Effect of processing, additives and vitamin B6 supplementation of Mucuna pruriens var cochinchinensis on broilers. J Trop \& Subtrop Agr 1: 227-237.

22. Valdivié M, Sampedro J, Cabezas L, Pérez N, Dieppa O. 2001. Comparación de híbridos nacionales e importados de pollos de engorde en Cuba. Crianza en jaulas en el verano. Rev Cub Cien Agric 35: 257-261. 